\title{
MEDICIÓN DE LA TRANSMISIÓN DE SÍMBOLOS EN PROGRAMAS CULTURALES
}

\author{
Guillermo Alfredo Zamacona Aboumrad* \\ DOI: https://doi.org/10.33571/revistaluciernaga.v11n22a5
}

\section{Resumen:}

En el artículo se presenta una manera de medir la transmisión simbólica en programas culturales. El estudio se fundamentó en la evaluación de 18 proyectos del Pacmyc programa mexicano con más de 25 años de implementación. Se concluye que al centrar la atención en la transmisión simbólica, y considerarla como factor a evaluar, el conjunto de herramientas que da forma al sistema de evaluación cultural de México se nutre de nuevas posibilidades permitiendo romper los esquemas de medición tradicionales e incorporando nuevas formas de evaluar el desempeño de los programas culturales públicos.

Palabras clave: Evaluación; política pública; programa cultural; transmisión simbólica.

Recibido. Junio 04, 2019

Aceptado. Junio 11, 2019

* Profesor investigador en la Universidad Anáhuac México. Doctor en América Latina Contemporánea por el Instituto Universitario de Investigación Ortega y Gasset en España, cuenta con una maestría en América Latina Contemporánea por la Universidad Internacional Menéndez Pelayo en España y un MBA por la Universidad Anáhuac México. Especializándose en la investigación cualitativa del sector cultural mexicano. De 2014 a la fecha ha participado en numerosos congresos internacionales sobre gestión cultural y políticas públicas. Su última publicación corresponde a la primera parte de este artículo: "Propuesta de indicadores para evaluar la sostenibilidad económica de proyectos culturales. Caso de estudio: Pacmyc", Gestión y Política Pública XXVIII: 1, Primer semestre (2019). Orcid: http://orcid.org/0000-0001-5270-6193 ; e-mail: guillermo.zamaconaa@anahuac.mx 


\title{
TRANSMISSION MEASURING ON SYMBOLS IN CULTURAL PROGRAMS
}

\author{
Guillermo Alfredo Zamacona Aboumrad* \\ DOI: https://doi.org/10.33571/revistaluciernaga.v11n22a5
}

\section{Abstract}

The article presents a mode of measuring symbolic transmission in cultural programs. The study was supported by the evaluation of 18 projects of the Pacmyc - a Mexican program with more than 25 years of implementation. It concludes that by focusing on symbolic transmission, and considering it as a factor to be evaluated, the set of tools that shape Mexico's cultural evaluation system is enriched with new possibilities allowing breaking the traditional measurement schemes and incorporating new ways to evaluate the performance of public cultural programs.

Keywords: Evaluation; public policy; cultural program; symbolic transmission.

Received. June 04, $2019 \quad$ Accepted. June 11, 2019 


\title{
MEDIÇÃO DA TRANSMISSÃO DE SÍMBOLOS EM PROGRAMAS CULTURAIS
}

\author{
Guillermo Alfredo Zamacona Aboumrad* \\ DOI: https://doi.org/10.33571/revistaluciernaga.v11n22a5
}

\section{Resumo}

O artigo apresenta uma forma de medir a transmissão simbólica em programas culturais. O estudo baseou-se na avaliação de 18 projetos do Pacmyc - um programa mexicano com mais de 25 anos de execução. Conclui que, ao centrar-se na transmissão simbólica e considerando-a como um factor a avaliar, o conjunto de ferramentas que moldam o sistema de avaliação cultural do México é alimentado por novas possibilidades que permitem quebrar os esquemas tradicionais de medição e incorporar novas formas de avaliar o desempenho dos programas culturais públicos.

Palavras chave: avaliação; política pública; programa cultural; transmissão simbólica.

Recebido. Junho 04, $2019 \quad$ Aceito. Junho 11, 2019

* Professor de investigação na Universidade de Anahuac, México. D. na América Latina Contemporânea pelo Instituto Universitario de Investigación Ortega y Gasset em Espanha, tem um mestrado em América Latina Contemporânea pela Universidad Internacional Menéndez Pelayo em Espanha e um MBA pela Universidad Anáhuac México. É especialista em investigação qualitativa do sector cultural mexicano. Desde 2014 até à data participou em numerosas conferências internacionais sobre gestão cultural e políticas públicas. A sua última publicação corresponde à primeira parte deste artigo: "Proposta de indicadores para avaliar a sustentabilidade económica dos projetos culturais". Estudo de caso: Pacmyc", Gestión y Política Pública XXVIII: 1, Primeiro Semestre (2019). 
La evaluación de los programas culturales resulta tan importante como la creación e implementación de políticas públicas ya que ésta es un paso más de un mismo proceso. Subirats (1989), en su libro Análisis de políticas públicas y eficacia de la administración, explica que este proceso está compuesto por una serie de pasos que comienzan con la identificación de un problema, continúa con la formulación de una política, sigue con la toma de decisiones sobre dicha política, procede a la implementación de programas y termina con la evaluación. Es posible encontrar estudios como eldeChartand \& McCaughey (1989), Zimmer \& Toepler (1999) o Zamorano (2014) que analizan, utilizando los pasos de este proceso, las políticas culturales. Desde hace aproximadamente tres décadas la dimensión cultural se fusionó con la dimensión evaluativa dando lugar a un nuevo campo de investigación (Castellano \& Alcívar, 2015).

La evaluación del sector cultural es una actividad relativamente nueva, sin embargo cada vez es más fácil encontrar estudios que proponen metodologías y herramientas de evaluación para este sector. Para Castellano \& Alcívar (2015), el enfoque neoliberal predomina en el campo de la evaluación cultural. Esta postura se observa en los informes de la Organización de Naciones Unidas [ONU] (2010, 2013), en los informes del Consejo Nacional de Evaluación de la Política de Desarrollo Social [Coneval] [1] -organismo encargado de evaluar los programas culturales mexicanos- y en las cuentas satélites de cultura.

Filosofía neoliberal, pone el foco en la medición de la eficiencia, el presupuesto, la cobertura y la rentabilidad de las acciones culturales.

Punto de vista desarrollista en éste predomina el estudio de las características de los beneficiarios de los programas. Este enfoque se puede observar en los documentos de la Agencia Española de Cooperación Internacional para el Desarrollo [Aecid] (2012), en los Indicadores Unesco de Cultura para el Desarrollo (2014).

También se encuentra la postura que se enfoca en la transmisión de los símbolos sociales, como la de la Carta Cultural Iberoamericana, para la generación de políticas públicas y programas culturales, muestra la importancia de los aspectos económicos y sociales de estos programas.

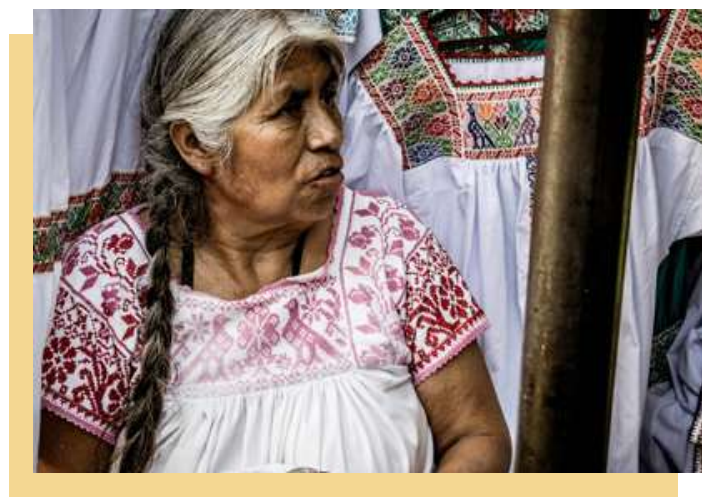

Este artículo se centra en la tercera postura. Para ello se analizaron 18 proyectos apoyados por el Pacmyc [2].

\section{El papel de la transmisión en los conceptos de cultura}

Santillán (2010, p. 35), señala que: "Todo tipo de política cultural (implícita o explícita) conlleva un concepto operativo de cultura (explícito o implícito) que sostiene una determinada línea de gestión o acción cultural y no otra." Por lo tanto, establecer el ángulo desde el cual se abarca el concepto de cultura es el primer paso en toda investigación sobre programas culturales.

- Perspectiva Institucional (Público ( Privado). Desde esta perspectiva se concibe a la cultura como el proceso que incluye un conjunto de símbolos 0 expresiones que son gestionados por los organismos culturales. Bajo esta línea, la institución administradora es la encargada de seleccionar los símbolos que "merecen" ser "administrados" y decide, por lo tanto, el conjunto de elementos que forman 
"la cultura". En México, asumen esta perspectiva la Secretaría de Cultura, los distintos programas culturales y artísticos, los museos, las escuelas de arte y los organismos que se dedican a promover las artesanías.

Para Nelly Richard (2005), la cultura se puede entender como un proceso que "se preocupa sobre todo por las dinámicas de distribución, recepción y producto a administrar" (p. 456). Throsby (1999), al comentar sobre la definición que la Comisión Mundial de Cultura y Desarrollo presentó en 1995 [3], resalta que ésta refiere a un "conjunto de actividades que incluyen a todas las acciones que se llevan a cabo en el interior de las llamadas 'industrias culturales'" (p. 6). Similar postura la respalda Jesús Martín-Barbero para quien la cultura se debe comprender "como proceso de comunicación regulado a un mismo tiempo por dos lógicas: la de las formas, o matrices simbólicas y la de los formatos industriales" (1993, p. 9).

- Perspectiva antropológica. Desde la antropología se asume la cultura como el proceso que incluye un conjunto de patrones que son adquiridos y transmitidos por los integrantes de un grupo social. Valentina Salvi menciona que "la concepción de la cultura como simbólica se apoya [...] en la noción weberiana del hombre como un animal suspendido en tramas de significaciones. Estas son tejidas colectivamente y dan lugar a la vida cultural" (2010, p. 24). Esta perspectiva enfatiza en que los sistemas culturales pueden ser el resultado o el condicionante de una acción; resalta que la cultura es un ciclo que se alimenta de sí mismo ( Kroeber \& Kluckhohn ,1952) (García Canclini, 1981) (Throsby, 1995; citado en Throsby, 1999) (Unesco, 2017). Salvi, reflexionando sobre las ideas de Geertz, comenta que "la cultura es un sistema de signos interpretables que han sido producidos por los agentes en el curso de su cotidianidad" (2010, p. 24).

- Perspectiva Artística. Esta clasificación relaciona la cultura con las bellas artes. Nelly Richard (2005) limita lo cultural a una "producción de formas y sentidos que se rige por instituciones y reglas de discurso especializadas, y que se manifiesta a través de obras" (p. 455).

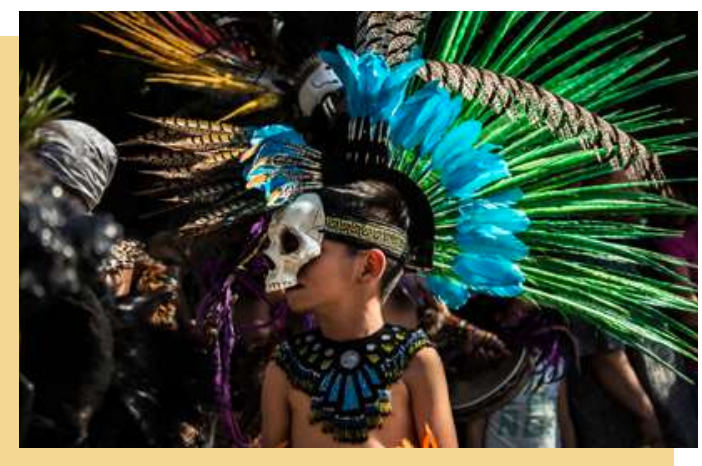

Throsby (2001) en su libro Economics and Culture, explica que existen dos formas de concebir el concepto de cultura; la primera, entendiéndola como un conjunto de actitudes, valores y costumbres que un grupo comparte; y la segunda, como actividades que se derivan de la educación o "iluminación" de la mente en contraposición a las actividades provenientes de habilidades técnicas. En este sentido, la cultura se enlaza directamente con las artes y la educación, y se considera como adjetivo que denota superioridad social e intelectual. En esta línea un grupo "elitista y estudiado" define los símbolos que forman la cultura.

- Perspectiva Estratégica. Desde este enfoque se entiende la cultura como recurso a ser considerado -y utilizadodentro de los programas económicos, sociales o políticos. George Yúdice resalta que la proliferación de argumentos en los foros donde se discuten proyectos tocantes a la cultura y al desarrollo locales, [...] han transformado lo que entendemos por el concepto de cultura y lo que hacemos en su nombre. (2003, p. 23), y agrega:

La desmaterialización característica de muchas nuevas fuentes de crecimiento económico[...] ly la mayor distribución de bienes simbólicos en el comercio mundial (filmes, programas de televisión, música, turismo, etc.) han dado a la esfera cultural un protagonismo mayor que en cualquier otro momento de la historia de la modernidad.

En otras palabras "lo simbólico" se vuelve significativo en muchas actividades vinculadas con el crecimiento económico al mismo tiempo que los bienes culturales 
comienzan a ser relevantes para este crecimiento. Dice Yúdice:

Se invoca la cultura cada vez más no sólo como un motor del desarrollo capitalista, [...] hay quienes aducen incluso que la cultura se ha transformado en la lógica misma del capitalismo contemporáneo, una transformación que ya está poniendo en tela de juicio nuestros presupuestos más básicos acerca de lo que constituye la sociedad humana. (2003, p.31)

Para Santillán (2010), la gran aportación de Yúdice, está en haber señalado que lo cultural pasó a ser operado de manera explícita por agentes de otras esferas (la económica, la política y la social) y que únicamente patenta cómo se pasa a operar desde lo simbólico y se intentan imponer ciertas prácticas y significados con mayor violencia simbólica y tecnológica.

Bourdieu (1984), menciona que cuando un individuo es capaz de reconocer y de crear las diferencias que existen en un campo social, y de aceptarlas como significativas, se empiezan a generar unidades sociales distintas. Sobre este proceso, Santillán comenta que cada comunidad crea un "universo de símbolos" que otorga sentido al estilo de vida:

Esto se visibiliza a través de diversos canales de comunicación [...] que posibilitan la construcción y transmisión [...] de: conocimientos, tecnologías, habilidades, formas de expresión artística, valores, ideas, imágenes, símbolos, mitos, representaciones, creencias y formas de actuar de esta sociedad. (2010, p. 44)

\section{Evaluación delosprogramas culturales en México}

En México el Consejo Nacional de Evaluación de la Política de Desarrollo Social - Coneval -, define al proceso de evaluación como el "análisis sistemático e imparcial de una intervención pública cuya

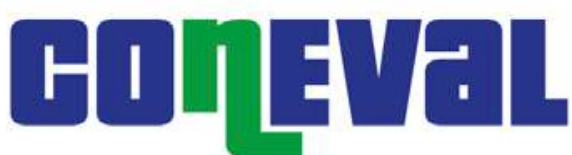

Consejo Nacional de Evaluación de la Política de Desarrollo Social

finalidad es determinar la pertinencia y el logro de sus objetivos y metas, así como la eficiencia, eficacia, calidad, resultados, impacto y sostenibilidad" (2013, p. 5). Las herramientas que utiliza este sistema para el análisis son:

Informes del Coneval: documentos que representan el estándar más alto en materia de evaluación de los programas culturales; encuestas de hábitos, prácticas y consumo cultural: herramientas que miden el movimiento del sector en su totalidad. Lo que brinda cuantiosa información ligada a la capacidad y al consumo cultural del país. Informes de evaluación de proyectos: establecen un vínculo entre la administración pública que busca recopilar información sobre los proyectos apoyados bajo sus diversos programas- y los beneficiarios. Cuenta satélite de la cultura en México: " [...] constituye la herramienta a través de la cual se presentan los agregados macroeconómicos y principales indicadores del sector, los cuales cuantifican los flujos generados por las actividades económicas asociadas con las prácticas culturales [...]" (Instituto Nacional de Estadística y Geografía [Inegi], 2014, p.1).

Si se analiza el contenido de dichas herramientas es posible encontrar información relacionada con: el consumo cultural (qué, quién, cómo y cuándo se consume), su cobertura (alcance geográfico y población beneficiada) y su gestión (cumplimiento de las normas y la eficiencia en el uso del presupuesto, principalmente). Si se comparan estos resultados con la clasificación de cultura que se mostró previamente, es posible resaltar que dichas herramientas no contemplan: 
El tipo de expresión o símbolo que se apoya. Los beneficios generados por la apropiación de dicha expresión - símbolo. La efectividad de su transmisión. La relevancia para los miembros involucrados.

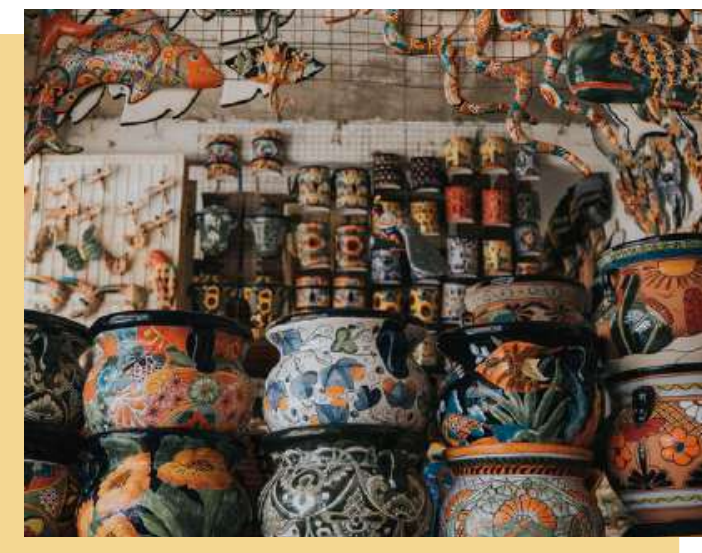

Como lo demuestran múltiples informes del Coneval, esta evaluación únicamente mide los aspectos administrativos del programa e ignora los resultados, beneficios y, más aún, la capacidad de transmisión de los símbolos contemplados.

Con la intención de ejemplificarbrevemente lo antes mencionado, a continuación, se muestra un pequeño análisis de El Informe de la Evaluación Específica de Desempeño 2014-2015 del Programa de Apoyo a las Culturas Municipales y Comunitarias [Pacmyc] [4]. En dicho informe se observa que:

- El resultado del programa debe ser la solución de un problema social y no solo la entrega de un apoyo; es decir, se busca un cambio en la sociedad, no entregar recursos para lograr el cambio:

"El Programa en 2014 y 2015 no identificó claramente el problema que buscaba atender". El objetivo principal se centró en la entrega de un bien (apoyo financiero) pero no en el cambio que se pretendía generar en la población objetivo; lo que debería haber sido lo fundamental según Nik Beta SC (2015, p. 9).

- Todos los análisis que se realizaron y los indicadores que se utilizaron se enfocaron en la medición del desempeño de las actividades realizadas dentro de las instituciones de gobierno. A pesar de que el informe establece que el fin del programa era "contribuir a procesos culturales comunitarios a través del apoyo financiero a proyectos culturales en la sociedad mexicana" (Nik Beta SC, 2015, p. 1).

En ningún momento se midió la intervención que se realizó sobre los procesos culturales o la forma en la que se promovió el desarrollo de las culturas populares; no se evaluaron las expresiones, un bien o un servicio cultural.

- No se realizaron otro tipo de evaluaciones debido a su alto valor financiero. El informe menciona: "El costo de la evaluación de impacto equivale a dejar de apoyar aproximadamente 100 proyectos y dado el presupuesto total del programa se pone en duda la factibilidad y necesidad de una evaluación de este tipo" (Nik Beta SC, 2015, p. 2).

Se entiende entonces que la forma en la que el Coneval evalúo este programas tuvo al menos estas dos limitantes: no evidenció, ni midió los diferentes resultados que arrojó el programa y no consideró el concepto de transmisión cultural.

\section{Pacmyc Querétaro 2012}

El Programa de Apoyo a las Culturas Municipales y Comunitarias (Pacmyc) es:

...una estrategia orientada a apoyar la recuperación y el desarrollo de la cultura popular, a través del financiamiento a proyectos que permitan estimular las iniciativas culturales de la sociedad.

Este programa se enfoca en "grupos interesados en recibir apoyo económico para desarrollar un proyecto cultural que fortalezca la identidad y procesos culturales de sus comunidades, en los espacios geográficos y simbólicos donde se desarrollan" (Dgcp, 2012). De acuerdo con el Diario Oficial de la Federación, que 
establece las Reglas de Operación del programa, su objetivo es:

"Contribuir al fortalecimiento de procesos culturales comunitarios, a promover y difundir el arte y la cultura como recursos formativos privilegiados para impulsar la educación integral, a través del apoyo financiero a proyectos culturales comunitarios que promuevan y difundan la diversidad de expresiones de la cultura popular" (Sep, 2014, p.5).

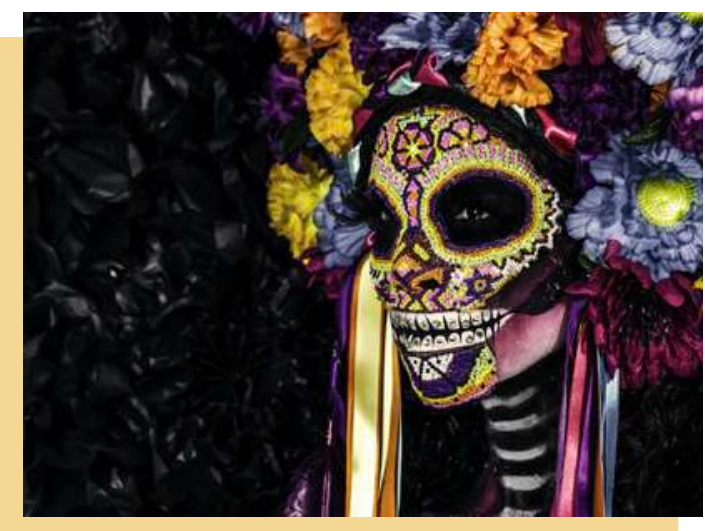

De forma específica, el Pacmyc pretende: Promover procesos y expresiones de las culturas populares a través del financiamiento de propuestas colectivas. Propiciar la participación de todos los órdenes de gobierno, así como de otras instancias sociales y privadas, en la integración de un fondo económico que busque apoyar proyectos de cultura popular (Sep, 2014, p.6).

De manera cronológica y resumida, el programa funciona así:

- El Pacmyc emite una convocatoria a nivel nacional invitando a "Portadoras y portadores de cultura popular que de forma colectiva tengan interés en desarrollar un proyecto cultural" (Sep, 2014, p.6).

- Los interesados envían sus proyectos.

- Los proyectos recibidos que cumplen con todos los requisitos participan en un proceso de selección.

- Un jurado plural e imparcial evalúa y califica cada proyecto presentado.

"Los jurados son elegidos por una
Comisión de Planeación y Apoyo a la Creación Popular (Cacrep) de cada entidad, la cual cuenta con representantes de la sociedad civil, así como de la instancia cultural en el estado y la federal" (Dgcp, 2012, p.52).

- Se anuncian los ganadores.

- A los ganadores se les apoya con un monto económico que varía según cada proyecto. El monto máximo es de una sola exhibición de 50,000 pesos mexicanos. [5]

Se decidió estudiar al Pacmyc como objeto de estudio debido a que el programa cumplía con todas las características necesarias para explorar los diversos resultados que un programa cultural podía generar. De forma específica, se seleccionaron los 18 proyectos apoyados por el Pacmyc Querétaro en la convocatoria de 2012 [6].

\section{Metodología}

Se utilizó como base metodológica la Teoría Fundamentada de Glaser y Strauss (1967) que permite generar teoría a partir del análisis de un caso en particular; y el Estudio de Caso (Coller, 2005 y Yin, 1994), que permite reflexiones a partir de un fenómeno en concreto. De igual forma, se utilizaron como referencia los análisis de Rouet (1989), Bustamante (2003) y Prosper (2009) quienes realizaron estudios utilizando los conceptos de cadena de producción y/o cadena de transmisión.

El estudio se dividió en dos fases: primero se utilizó un enfoque cualitativo para conocer los resultados generados por el Pacmyc; y en segundo lugar se utilizó un enfoque cuantitativo para elaborar y comprobar una serie de indicadores.

El objetivo último de la investigación fue elaborar una herramienta que permitiera conocer en qué medida el Pacmyc apoya la transmisión simbólica con el objetivo de comprobarla hipótesis: los programas culturales públicos no apoyan de manera central la transmisión de los símbolos culturales que contemplan. 
Se definió como variable dependiente "el apoyo a la transmisión de los símbolos" y como variable independiente "las actividades realizadas en los proyectos del Pacmyc". Acorde a la metodología utilizada [7], las herramientas de investigación que se utilizaron fueron las siguientes:

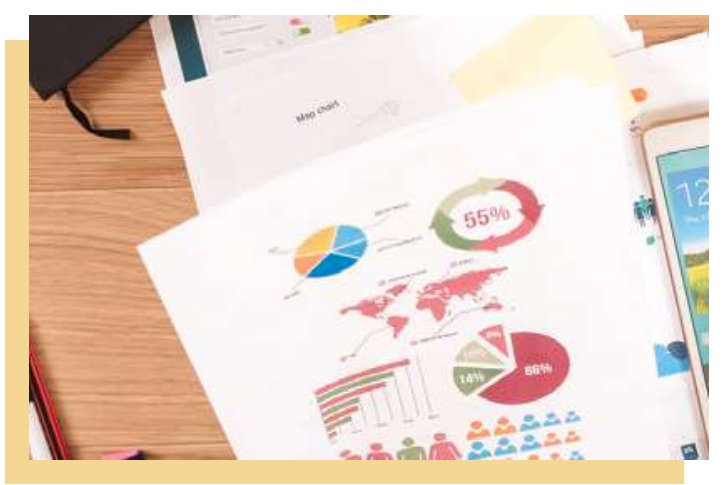

- Entrevista en profundidad semiestructurada: El uso de esta herramienta permitió conocer los resultados obtenidos por el Pacmyc y comprender las razones por las cuales los beneficiarios realizan proyectos culturales. Se entrevistó a: diecisiete a beneficiarios [8], dos promotores culturales, al Coordinador del Pacmyc, al Director de la Unidad Regional de Querétaro, al responsable de actividades operativas a nivel nacional, al coordinador del programa a nivel nacional, a la coordinadora del Centro de Información y Documentación Alberto Beltrán.

- Análisis de documentos: se estudiaron diversos libros y documentos, tanto públicos como privados, con el objetivo de profundizar en la comprensión del programa estudiado. Esta herramienta fue fundamental para conocer cómo funcionaba el Pacmyc en años anteriores, comprender su gestión actual y entender la composición y evaluación de los proyectos.

- Observación no participante: se optó por esta herramienta debido a que era necesario conocer la forma en la que el personal del Pacmyc se relacionaba con los beneficiarios del programa. El objetivo era conocer la influencia que tienen los promotores culturales y el Director de la Unidad Regional sobre los proyectos elaborados. Esta herramienta se utilizó durante una sesión de promoción del programa. Se seleccionó esta actividad debido a que es el momento en el que hay un mayor número de personas interesadas en el programa interactuando con los miembros del Pacmyc. Es el instante en el que más pueden influir los promotores culturales en la elaboración de los proyectos.

- Grupo de discusión: se realizó un grupo de discusión para conocer al Pacmyc y para entender las múltiples ideas que existen alrededor de los proyectos que se apoyan [9].

\section{Resultados}

\section{- Primera fase}

Se estudió a profundidad el funcionamiento del programa y se indagó en la composición de los proyectos apoyados: se analizaron las actividades que estos incluían, sus resultados y los beneficios percibidos por los participantes.

El análisis de los proyectos apoyados por el Pacmyc reflejó una forma de clasificarlos con base en la transmisión de los símbolos de cada proyecto. Es decir, fue posible identificar tareas dentro de los proyectos que se relacionaban con las siguientes actividades:

\section{- Creación, interpretación o} reinterpretación: idear una expresión que no existía, formar algo nuevo cambiando las características de algo que ya existía, o concebir y ordenar, con base en términos propios, el significado de una idea, concepto o expresión.

- Reproducción: ejecutar o desarrollar una expresión existente.

- Difusión: acto de comunicar o propagar una idea, concepto, expresión o mensaje.

- Participación: intervención de forma activa o pasiva en una idea, concepto, actividad o expresión (por ejemplo, se puede participar como receptor de un mensaje, como invitado en una fiesta municipal o como actor en una obra teatral).

- Apropiación: hecho de tomar para sí, a modo de hacer suya, una idea, concepto, expresión o mensaje. 
Al ordenar estas actividades, es posible elaborar una cadena de actividades que tiene como fin último la transmisión de los símbolos contemplados dentro de los proyectos. De forma gráfica, estas actividades se pueden explicar de la siguiente forma:

\section{Ilustración 1: Cadena de transmisión cultural}

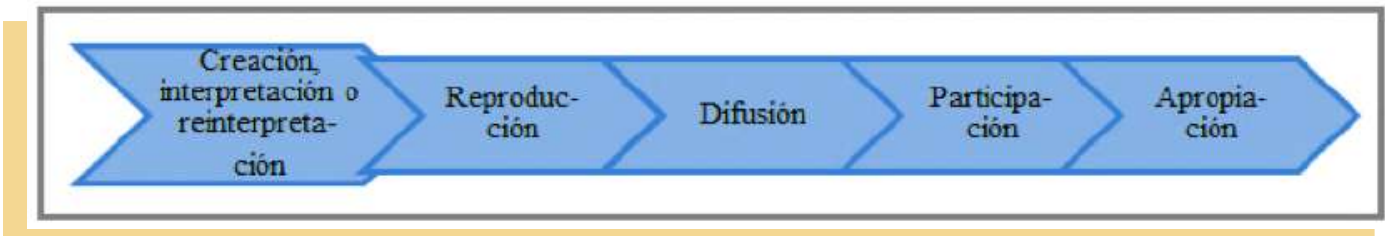

Fuente: Elaboración propia con base en los autores citados y los resultados de la investigación

Esta "cadena de transmisión cultural" presupone que cuando los espectadores o participantes en una expresión se apropian de los mensajes implícitos, son capaces de reelaborarlos y retransmitirlos convirtiéndose en creadores de nuevas expresiones culturales o en emisores de símbolos previamente existentes.

Con base en esta clasificación, y con la intención de profundizar en el estudio de los programas culturales, fue necesario conocer la cantidad de expresiones que el Pacmyc - Querétaro consideró en el 2012. Debido a que un proyecto podía contener múltiples expresiones o elementos simbólicos fue necesario fragmentar cada uno de los proyectos; este ejercicio arrojó un total de 24 expresiones culturales consideradas.

Después de fragmentar los proyectos estudiados y detectar las expresiones consideradas, se les relacionaron las actividades antes mencionadas con las 24 expresiones detectadas. Se encontraron proyectos que únicamente reproducían expresiones o proyectos que reproducían, difundían y promovían la participación de la sociedad en una misma expresión. A continuación, se presenta un gráfico que relaciona las expresiones estudiadas con las actividades propuestas.

Gráfico 1.

Relación de expresiones culturales con actividades de transmisión

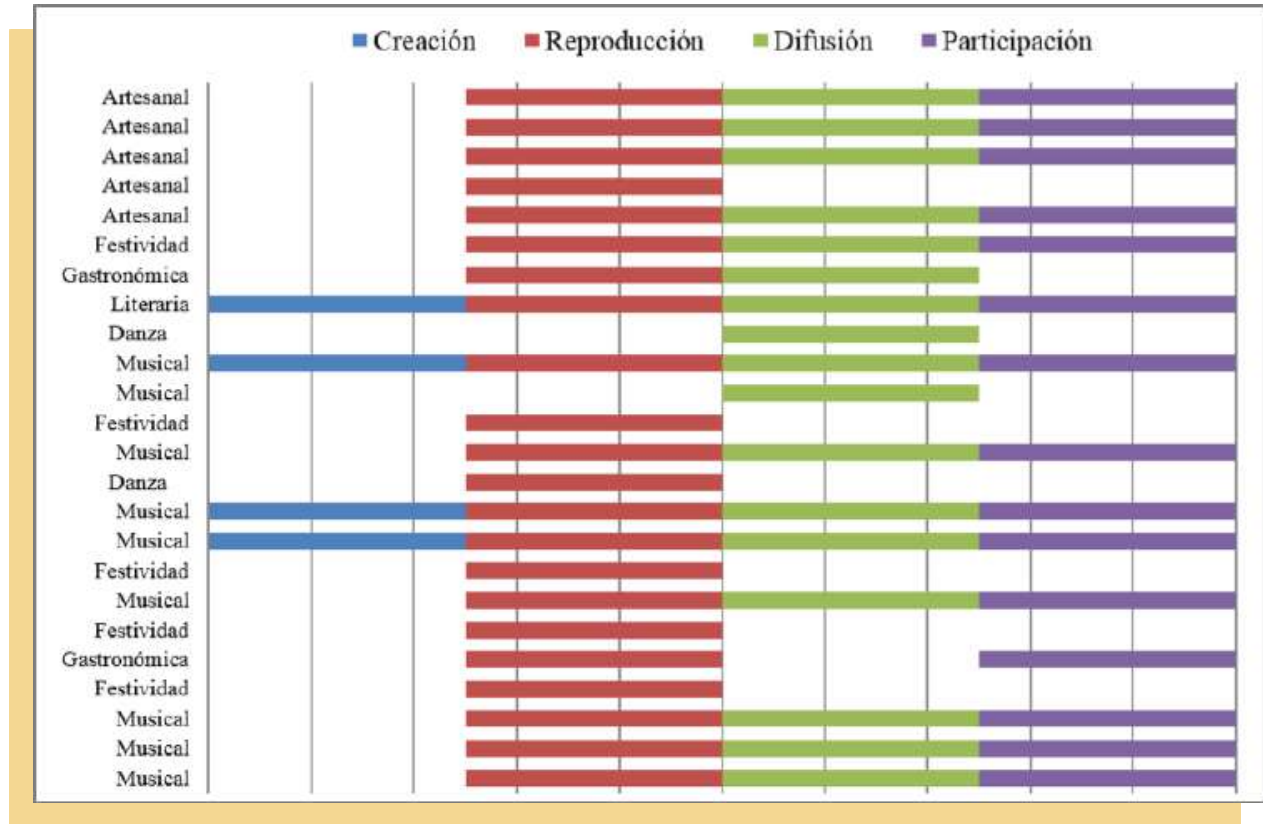

Fuente: Elaboración propia 
Este análisis es fundamental ya que despliega los resultados culturales de manera aislada y muestra lo que sucede con cada una de las expresiones apoyadas; es decir, permite evaluar lo que sucede con la transmisión de las expresiones culturales. De los datos analizados se puede resumir que:

- Las actividades culturales realizadas en los proyectos estudiados centraron sus esfuerzos en la reproducción de expresiones

- Pocas expresiones incluyeron actividades creativas

- Hay más expresiones reproducidas que expresiones que consideraron la participación de la sociedad

A modo de conclusión, vale la pena resaltar que este análisis permite relacionar las actividades de los proyectos estudiados con las actividades de la cadena de transmisión cimentando las bases para generar indicadores culturales que permitan comprobar la hipótesis planteada.

\section{- Segunda Fase}

Durante la segunda fase, se utilizó como base los resultados de la sección anterior, se desarrollaron indicadores con el objetivo de conocer la capacidad de transmisión de los proyectos culturales. Estos se dividieron en dos grupos (expresiones culturales atendidas y transmisión simbólica), estos representan una nueva herramienta de evaluación que proporciona información sobre la transmisión cultural de los programas.

Este apartado muestra, en primer lugar, el conjunto de indicadores creado $y$, en segundo, el ejercicio con el cual se comienza a probar su efectividad.

\section{- Creación de indicadores}

Indicadores sobre expresiones culturales atendidas. Este primer grupo está integrado por seis indicadores y su objetivo es conocer la cantidad de expresiones que fueron consideradas por el programa y la forma en la que este las apoyó.
1. Nombre del indicador: Proporción de expresiones culturales que se encuentran en la lista del patrimonio cultural material e inmaterial del estado que se vieron beneficiadas de forma directa [10] por el programa. Método de cálculo: (Expresiones que se encuentran en el listado de patrimonio del estado apoyadas económicamente en el año t/total de expresiones culturales apoyadas en el año t) x100. Unidad de medida: Porcentaje. Frecuencia de medición del indicador: Anual.

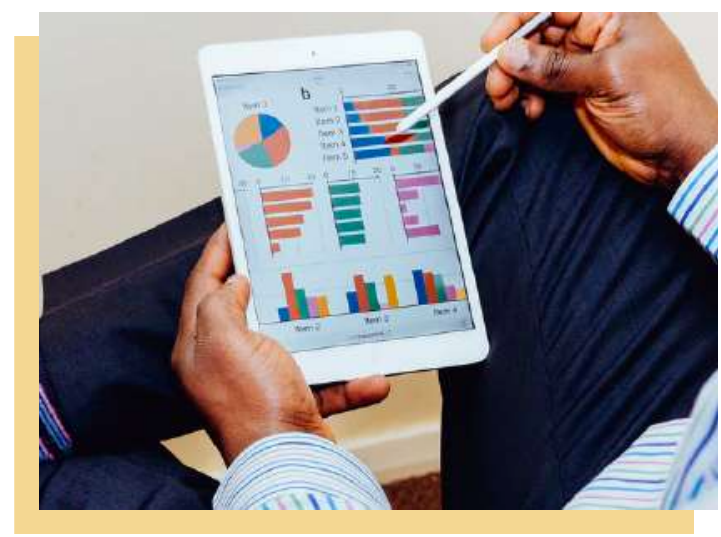

2. Nombre del indicador: Proporción de expresiones culturales que se encuentran en la lista del patrimonio cultural material e inmaterial del estado que se vieron beneficiadas de forma indirecta [11] por el programa. Método de cálculo: (Expresiones que se encuentran en el listado de patrimonio del estado que fueron contempladas pero que no recibieron apoyo económico en el año t/total de expresiones culturales apoyadas en el año t) x100. Unidad de medida: Porcentaje. Frecuencia de medición del indicador: Anual.

3. Nombre del indicador: Proporción de expresiones culturales que no se encuentran en la lista del patrimonio cultural material e inmaterial del estado pero que se vieron beneficiadas de forma directa por el programa. Método de cálculo: (Expresiones que no se encuentran en la lista del patrimonio cultural pero que fueron apoyadas en el año t/total 
de expresiones culturales apoyadas en el año t) x100. Unidad de medida: Porcentaje. Frecuencia de medición del indicador: Anual.

4. Nombre del indicador: Proporción de expresiones culturales que no se encuentran en la lista del patrimonio cultural material e inmaterial del estado pero que se vieron beneficiadas de forma indirecta por el programa. Método de cálculo: (Expresiones que no se encuentran en la lista del patrimonio cultural y que fueron contempladas sin apoyo económico en el año t/total de expresiones culturales apoyadas en el año t) x100. Unidad de medida: Porcentaje. Frecuencia de medición del indicador: Anual.

5. Nombre del indicador: Proporción de expresiones culturales extranjeras a la comunidad que se vieron beneficiadas de forma directa por el programa. Método de cálculo: (Expresiones extranjeras apoyadas económicamente en el año t/total de expresiones culturales apoyadas en el año t) x100. Unidad de medida: Porcentaje. Frecuencia de medición del indicador: Anual.

6. Nombre del indicador: Proporción de expresiones culturales extranjeras a la comunidad que se vieron beneficiadas de forma indirecta por el programa. Método de cálculo: (Expresiones extranjeras contempladas sin apoyo económico en el año t/total de expresiones culturales apoyadas en el año $t$ ) x100. Unidad de medida: Porcentaje. Frecuencia de medición del indicador: Anual.

Estos indicadores muestran la relación que existe entre lo que se apoya (expresiones - elementos simbólicos) y la forma de apoyarlo. Como se puede intuir, esta batería de indicadores utiliza la lista del patrimonio cultural del estado con el propósito de detectar si 'lo apoyado' es parte del patrimonio estatal, si proviene de fuera o si es una expresión local 'menos popular'. De igual forma, permite saber si 'lo apoyado' obtuvo recursos económicos provenientes del Pacmyc o si simplemente fue considerado en el proyecto.

La principal ventaja de estos indicadores es que permiten conocer hacia dónde se dirigen los recursos del programa estudiado.

\section{- Indicadores sobre la transmisión simbólica}

Este segundo grupo tiene por objetivo obtener información sobre la transmisión de las expresiones culturales apoyadas. Los nueve indicadores que lo componen utilizan como base la cadena de transmisión y, debido a que cada proyecto podría incorporar más de una expresión, se enfocan en expresiones consideradas en lugar de proyectos apoyados.

1. Nombre del indicador: Proporción de expresiones culturales que contemplan actividades de creación. Método de cálculo: (Expresiones creadas en el año t/ total de expresiones apoyadas en el año t) x100. Unidad de medida: Porcentaje. Frecuencia de medición del indicador: Anual.

2. Nombre del indicador: Proporción de expresiones culturales que contemplan actividades de reproducción. Método de cálculo: (Expresiones reproducidas en el año t/total de expresiones apoyadas en el año t) x100. Unidad de medida: Porcentaje. Frecuencia de medición del indicador: Anual.

3. Nombre del indicador: Proporción de expresiones culturales que contemplan actividades de difusión. Método de cálculo: (Expresiones difundidas en el año t/total de expresiones apoyadas en el año $t$ ) $x 100$. Unidad de medida: Porcentaje. Frecuencia de medición del indicador: Anual.

4. Nombre del indicador: Proporción de expresiones culturales que 
contemplan espectadores en sus actividades. Método de cálculo: (Expresiones que contemplaron actividades con espectadores en el año $t /$ total de expresiones apoyadas en el año t) $x 100$.

Unidad de medida: Porcentaje. Frecuencia de medición del indicador: Anual.

5. Nombre del indicador: Proporción de expresiones culturales que contemplaron todos los eslabones de la cadena de transmisión. Método de cálculo: (Expresiones culturales que contemplaron todos los eslabones de la cadena de transmisión en el año $t /$ total de expresiones culturales apoyadas en el año t) x100. Unidad de medida: Porcentaje. Frecuencia de medición del indicador: Anual.

Este segundo conjunto de indicadores otorga información sobre la transmisión de las expresiones apoyadas ya que relaciona las actividades realizadas en los proyectos con los eslabones de la cadena de transmisión. Estos cinco indicadores son, quizá, los más importantes de todo el grupo ya que demuestran la forma en la que el Pacmyc interviene en la transmisión de los símbolos considerados.

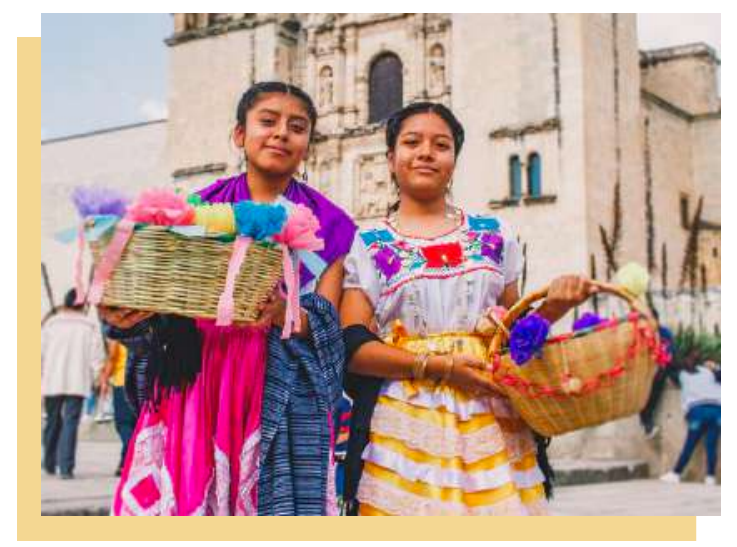

Para finalizar, el foco de los cuatro últimos indicadores deja de estar en las actividades apoyadas y centra su atención en el papel de la población.

6. Nombre del indicador: Variación porcentual de creadores. Método de cálculo: [(Creadores en el año t/ creadores en el año t-1)-1] x100. Unidad de medida: Porcentaje. Frecuencia de medición del indicador: Anual.

7. Nombre del indicador: Variación porcentual de reproductores. Método de cálculo: [(Reproductores en el año t/reproductores en el año t-1)-1] x100. Unidad de medida: Porcentaje. Frecuencia de medición del indicador: Anual.

8. Nombre del indicador: Variación porcentual de promotores. Método de cálculo: [(Promotores en el año t/promotores en el año t-1)-1] x100. Unidad de medida: Porcentaje. Frecuencia de medición del indicador: Anual.

9. Nombre del indicador: Variación porcentual de espectadores. Método de cálculo: [(Espectadores en el año t/espectadores en el año t-1)-1] x100. Unidad de medida: Porcentaje. Frecuencia de medición del indicador: Anual.

Estos últimos indicadores revelan el número de personas que entraron en contacto con las expresiones beneficiadas y visibilizan el rol que estos desempeñaron.

Posterior a la creación de los indicadores, se validó su eficiencia aplicándolos a los proyectos financiados por el Pacmyc en el Estado de Querétaro en la convocatoria de 2012. A continuación los resultados encontrados:

\section{Comprobación de los indicadores}

- Proporción de expresiones culturales que se encuentran en la lista del patrimonio cultural material e inmaterial del estado que se vieron beneficiadas de forma directa por el programa: $35.9 \%$.

- Proporción de expresiones culturales que se encuentran en la lista del patrimonio cultural material e inmaterial del estado que se vieron beneficiadas de forma indirecta por el programa: $33.33 \%$.

- Proporción de expresiones culturales que no se encuentran en la 
lista del patrimonio cultural material e inmaterial del estado pero que se vieron beneficiadas de forma directa por el programa: $20.51 \%$.

- Proporción de expresiones culturales que no se encuentran en la lista del patrimonio cultural material e inmaterial del estado pero que se vieron beneficiadas de forma indirecta por el programa: $7.69 \%$.

- Proporción de expresiones culturales extranjeras a la comunidad que se vieron beneficiadas de forma directa por el programa: $2.56 \%$.

- Proporción de expresiones culturales extranjeras a la comunidad que se vieron beneficiadas de forma indirecta por el programa: $0 \%$.

Esta primera aplicación revela que el 35.9\% de las expresiones que utilizan recursos económicos del Pacmyc pertenece a la lista del patrimonio del Estado de Querétaro. Si a este dato se le agregan las expresiones de la lista que fueron contempladas en los proyectos pero que no recibieron recursos económicos, el porcentaje aumenta casi al doble $-69.24 \%$.

En otras palabras, el $30.76 \%$ de las expresiones consideradas -hayan recibido recursos económicos o no- no pertenece a la lista del patrimonio del estado. Como se puede observar, la relevancia de estos datos recae en la centralidad de lo cultural y en la identificación del destino final del apoyo gubernamental.

Comprobación de los indicadores sobre la transmisión simbólica

- Proporción de expresiones culturales que contemplan actividades de creación: $16.67 \%$.

-Proporción de expresionesculturales que contemplan actividades de reproducción: $91.67 \%$.

- Proporción de expresiones culturales que contemplan actividades de difusión: $70.83 \%$.

- Proporción de expresionesculturales que contemplan espectadores en sus actividades: $62.5 \%$.

- Proporción de expresiones culturales que contemplaron todos los eslabones de la cadena de transmisión: $12.5 \%$.
Gráfica 2.

Eslabones de la cadena transmisión simbólica apoyados por el Pacmyc

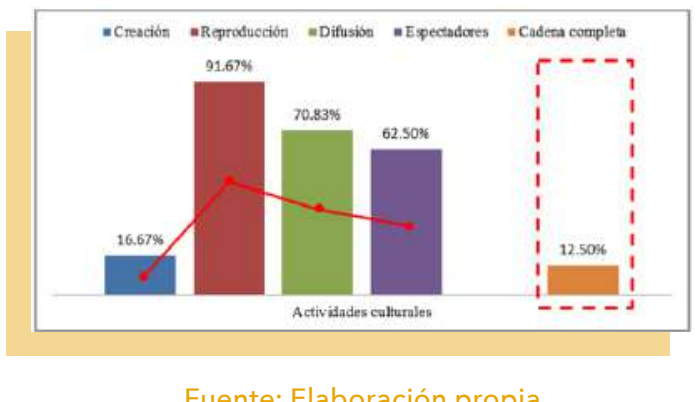

Como se muestra en el gráfico anterior, el $91.67 \%$ del total de las expresiones fueron reproducidas, el $70.83 \%$ fueron difundidas y el $62.5 \%$ se incluyeron en actividades en donde participó la sociedad; resulta interesante que únicamente el $16.6 \%$ de las expresiones contempladas incluyó actividades de creación.

Estos indicadores reflejan el tipo de actividades que el programa apoya y demuestran que el Pacmyc - Querétaro 2012 centró sus esfuerzos en la reproducción de expresiones culturales dejando en segundo plano la difusión y la participación de la sociedad.

El resultado más relevante es, quizás, que $37.5 \%$ de las expresiones consideradas no se incluyeron en actividades que involucraron al resto de la sociedad. Este dato se traduce en una baja participación de la sociedad y en una baja apropiación de las expresiones consideradas.

Para finalizar, se presentan los resultados del último conjunto de indicadores:

- Variación porcentual de creadores: No aplica. 57 creadores contemplados.

-Variación porcentual de reproductores: No aplica. 274 reproductores contemplados.

- Variación porcentual de promotores: No aplica. 175 promotores contemplados.

- Variación porcentual de espectadores: No aplica. 4,709 espectadores contemplados. 
Es posible notar que mientras 274 personas reprodujeron las expresiones consideradas únicamente 175 personas las difundieron. De igual forma, es posible observar que en promedio cada persona que reprodujo una expresión impactó únicamente en $17(4,709 / 274)$ personas. Estos números reafirman el resultado de los indicadores anteriores y vuelven a reflejar la poca capacidad de transmisión que tiene el programa.

\section{Conclusiones, limitantes y futuras líneas de investigación}

Existen diversos estudios sobre la creación e implementación de las políticas culturales en Latinoamérica; están los que hacen comparativos entre países, los que se adentran en las particularidades de la región, los que detallan su creación e implementación, entre otros; pero son pocos los que estudian a las políticas desde la evaluación de sus programas.

Al analizar las investigaciones que se centran en la evaluación de las políticas culturales o en las actividades que de ellas emanan, resulta evidente que ninguna de estas considera la transmisión de las expresiones culturales; es decir, ninguna evalúa qué es lo que sucede con estas expresiones durante o después de la ejecución de dichas actividades. En este sentido, es posible destacar dos líneas que pueden y deben guiar futuras investigaciones: la evaluación como parte central en el debate de las políticas culturales, la transmisión simbólica como objeto de estudio

El análisis bibliográfico y los resultados del estudio del caso que aquí se presentaron permiten asegurar que la transmisión simbólica, a pesar de ser un factor que se encuentra en la médula del sector cultural, no se considera en las herramientas de evaluación de México. Actualmente los métodos de evaluación olvidan que el proceso de transmisión se encuentra inmerso en todas las áreas del sector cultural (independientemente de la definición que se utilice) y que es la base de cualquier programa o actividad que se desarrolla bajo este ámbito. Esta omisión, poco justificada desde el punto de vista teórico, restringe la información que los evaluadores obtienen por lo que se entregan análisis limitados.

Al centrar la atención en la transmisión simbólica, y considerarla como factor a evaluar, el conjunto de herramientas que da forma al sistema de evaluación cultural de México se nutre de nuevas posibilidades permitiendo romper los esquemas de medición tradicionales e incorporando nuevas formas de evaluar el desempeño de los programas culturales públicos.

Esta condición permite centrar miras en los aspectos propios de la cultura y dejar en segundo plano las variables de consumo, cobertura y gestión. Más importante aún, esta propuesta cuestiona la propia gestión cultural y expone nuevas variables para la creación de políticas culturales. De igual forma, el proceso de transmisión como variable central exige a los investigadores incluir nuevos conceptos en sus proyectos y cuestionar las variables que se utilizan en la actualidad.

En este sentido, es posible concluir que la hipótesis planteada en un inicio se comprueba de manera parcial ya que el $12.5 \%$ de las expresiones consideradas en el Pacmyc Querétaro 2012 síse involucraron en todas las actividades necesarias para garantizar su transmisión.

Por último, es importante mencionar que los indicadores que aquí se presentan únicamente constituyen una primera propuesta sobre cómo evaluar el proceso de transmisión, también es cierto que invitan a un sinfín de posibilidades en este campo. Esta propuesta abre el debate sobre la posición que ocupa el proceso de transmisión en las políticas culturales y cuestiona el verdadero objetivo de todo el sector. 


\section{Bibiografía}

AECID [Agencia Española de Cooperación Internacional para el Desarrollo]. (2012). Cómo evaluar proyectos de cultura para el desarrollo: Una aproximación metodológica a la construcción de indicadores. Madrid, España: AECID/MAEC.

Álvarez Díaz, A. E. (1992). Análisis de Políticas Públicas. Caracas, Venezuela: CLAD.

Bourdieu, P. (1984). Sociología y cultura. Paris, Francia: Les Editions de Minuit.

Bustamante, E. (2003). Las industrias culturales, entre dos siglos. En E. Bustamante (Coord.), Hacia un nuevo sistema mundial de comunicación. Las industrias culturales en la era digital (pp. 19-38). Barcelona, España: Gedisa.

Castellano, J. M. \& Alcívar, C. E. (2015). Percepción social de la cultura en el cantón Machalá, Ecuador. Culturales, Época II, III(2), 113-149. Recuperado de https://bit.ly/3c4EvHp

Chartrand, H.H. \& McCaughey, C. (1989). The Arm's Length Principle and the Arts: An International Perspective-Past, Present and Future. En: M. C. Cummings \& J. M. Schuster (Eds.) Who's to Pay for the Arts?: The International Search for Models of Support. 43-80. Nueva York, Estados Unidos de América: American Council for the Arts.

Coller, X. (2005). Estudios de Casos. (Cuadernos Metodológicos, 30). Madrid, España: CIS.

Consejo Nacional de Evaluación de la Política de Desarrollo Social. (2013). Evaluación Integral del Desempeño de los Programas Federales Dirigidos a la Cultura 2012-2013. Recuperado de: https://bit.ly/2LX2KwC

----- (2017). ¿Quiénes somos? Misión y visión. Recuperado de: https://bit.ly/3grWRFN

Dirección General de Culturas Populares (2012) Convocatoria para participar en el PACMYC 2012. México: CONACULTA. García Canclini, N. (1981). Las culturas populares en el capitalismo. La Habana, Cuba: Casa de las Américas.
Glaser, B.G. y Straus, A.L. (1967). The Discovery of Groundesd Theory: strategies for qualitative research. Nueva York, Estados Unidos de América: Transaction Publishers.

Instituto Nacional de Estadística y Geografía. (2014). Sistema de Cuentas Nacionales de México.

Cuenta satélite de la cultura de México 2008-2011. Recuperado de: https://bit.ly/2yAaPnT

Kroeber A. L. y Kluckhohn C. (1952). Culture. A Critical Review of Concepts and Definitions. Massachusetts, Estados Unidos de América: Harvard University.

Martin-Barbero, J. (1993). Industrias Culturales: Modernidad e Identidad. Revistes Catalanes amb Accés Obert, 15, 9-20. Recuperado de: https://bit.ly/36zwpVT

NIK BETA SC. (2015). Informe de la Evaluación Específica de Desempeño 2014 - 2015. Valoración de la información de desempeño presentada por el programa. Programa de Apoyo a las Culturas Municipales y Comunitarias (PACMYC). Recuperado de: https://bit.ly/3c5LwYg

Organización de las Naciones Unidas. (2010). The Creative Economy Report 2010. Nueva York, Estados Unidos de América: UN.

(2013). The Creative Economy Report 2013 Special Edition. Nueva York, Estados Unidos de América: UN/ UNDP/UNESCO.

Organización de las Naciones Unidas para la Educación, la Ciencia y la Cultura. (2014). Indicadores UNESCO de cultura para el desarrollo. Manual metodológico. Francia: UNESCO/Diversidad de las Expresiones Culturales/AECID.

(2017). Página de Sectores de Trabajo. Líneas Generales. Recuperado de: https://bit.ly/2X3RAMV

PACMYC. (s.f.). Metodología 19992000. Ciudad de México, México: CONACULTA. 
Prosper, M. (2012). La cara oculta de la Edición. Madrid, España: Trama Editorial. Richard, N. (2005). Globalización Académica, Estudios Culturales y Crítica Latinoamericana. En D. Mato (Coord.), Cultura, Política y Sociedad. 455-470. Buenos Aires, Argentina: CLACSO.

Rouet, F. (1989). Economie et Culture. Industries Culturelles, III.

Salvi, V. (2010). Cultura y Poder. Los Bienes Culturales como Símbolos de Estratificación Social. En O. Moreno (Coord.), Artes e Industrias Culturales. 23-34. Buenos Aires, Argentina: EDUNTREF.

Santillán Güemes, R. (2010). Hacia un Concepto Operativo de Cultura. En O. Moreno (Coord.), Artes e Industrias Culturales. 35-49. Buenos Aires, Argentina: EDUNTREF.

Secretaria de cultura. Dirección General de Culturas Populares. Programas. PACMYC. Recuperado el 9 de mayo de 2016, de: https://bit.ly/3eebU3U

Secretaría de Educación Pública. (2014). ACUERDO número 14/12/14 por el que se establece la Unidad de Seguimiento de Compromisos e Instrucciones Presidenciales en el Sector Educativo. Diario Oficial de la Federación, 22 de diciembre 2015, quinta sección. Recuperado de: https://bit.ly/2ZzYET8

------ (2014). ACUERDO número $14 / 12 / 14$ por el que se establece la Unidad de Seguimiento de Compromisos e Instrucciones Presidenciales en el Sector Educativo. Diario Oficial de la Federación, 22 de diciembre 2015, quinta sección.

Subirats, I. (1992). Análisis de Políticas Públicas. Madrid, España: MAP.

Throsby, D. (1999). Capital Cultural. Journal of Cultural Economics, 23, 3-12. Recuperado de: https://bit.ly/2ZzYHOO

(2001). Economics and Culture.

Reino Unido: Cambridge University

Press.

Yin, R. K. (1994). Case Study Research: Design and methods. $2^{\mathrm{a}}$ Edición. Londres, Reino Unido: SAGE.

Yúdice, G. (2003). El Recurso de la Cultura: Usos de la Cultura en la Era Global. Barcelona, España: Gedisa.

Zamorano, M. M.; Rius Ulldemolins, J. y Klein, R. (2014). ¿Hacia un modelo sudamericano de política cultural? Singularidades y convergencias en Uruguay, Paraguay y Chile en el siglo XXI. Revista Europea de Estudios Latinoamericanos y del Caribe. 96, abril 2014, 5-34.

Zimmer, A. y Toepler, S. (1999). The Subsidized Muse: Government and the Arts in Western Europe and the United States. 23(1), 33-49, Journal of Cultural Economics. Recuperado de: https://bit.ly/2TJs5yp

\section{Notas}

[1] El Coneval, tiene por misión "medir la pobreza y evaluar los Programas y la Política de Desarrollo Social para mejorar sus resultados y apoyar la rendición de cuentas" (Coneval, 2017). Para mayores referencias pueden consultarse los Informes de Evaluación que se encuentran en la página oficial del Coneval: http://www.coneval.org.mx/

[2]Programa de Apoyo a las Culturas Municipales y Comunitarias [Pacmyc]. Se desarrolla bajo la Dirección General de Culturas Populares de la Secretaría de Cultura. Es un programa con más de 30 años de trayectoria que ha financiado más de 23,000 proyectos culturales.
[3] El autor menciona que en el documento de la Comisión existe un intento por obtener una definición de cultura utilizando el contexto específico del desarrollo económico; sin embargo, la Comisión se resiste a no tratar el término de una manera general abarcando el sentido más amplio de la palabra.

[4] Informe realizado por el Coneval; éstos informes son los únicos documentos públicos que muestran la forma de medir los resultados del Pacmyc.

[5]En la edición del 2016 el monto máximo fue, por primera vez, de 60,000 pesos mexicanos. 
[6]Se seleccionó el Estado de Querétaro debido a que presentaba buena disposición a proporcionar información, poseía una masa crítica de proyectos apoyados y las características sociodemográficas y culturales del estado eran idóneas para el estudio. Por otro lado, se seleccionó la edición del 2102 por la disponibilidad de datos en el momento de la investigación de campo.

[7]Rodríguez, Gil y García, las fuentes de la Teoría Fundamentada "son las entrevistas y las observaciones de campo, así como los documentos de todo tipo (...) y las grabaciones audiovisuales" (1999, p.48).

[8]No fue posible entrevistar a uno de los beneficiarios debido a que no se pudo localizar; sin embargo, el análisis se realizó utilizando la información de su expediente.
[9] También se realizó un pequeño grupo de discusión con los miembros de la Unidad Regional del Estado de Querétaro con el objetivo de profundizar en cuestiones que resultaban necesarias. De igual forma, se realizó un grupo de discusión con miembros del programa a nivel nacional con el objetivo de entender el funcionamiento general del Pacmyc.

[10] Expresiones que fueron parte del proyecto y que se incluyeron en actividades financiadas directamente por el Pacmyc.

[11]Expresiones que fueron parte del proyecto pero que no se incluyeron en las actividades financiadas por el Pacmyc.

\section{Para citar:}

Zamacona, G. (2019). Medición de la transmisión de símbolos en programas culturales. Revista Luciérnaga Comunicación. Vol. 11, Núm. 22. Pp 112-129.

Doi: https://doi.org/10.33571/revistaluciernaga.v11n22a5

OJS. https://revistas.elpoli.edu.co/index.php/luc/issue/archive 